\title{
An Inner Medullary Concentrating Process Actuated by Renal Pelvic/Calyceal Muscle Contractions: Assessment and Hypothesis
}

\author{
G.G. Pinter ${ }^{\mathrm{a}}$ J.L. Shohet ${ }^{\mathrm{b}}$

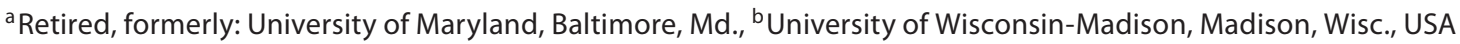

\section{Key Words}

Hyaluronan - Extravasated plasma albumin •

Gibbs-Donnan effect

\begin{abstract}
We propose a mechanism of an inner medullary concentrating process in which water extraction is accomplished by a colloid osmotic mechanism and hydrostatic pressure. There are 3 essential features of the proposal: 1. the fluid compartmental structure of the inner medullary interstitium: owing to molecular exclusion, negatively charged macromolecules, i.e. hyaluronan and extravasated plasma albumin form separate compartments (the HA and the EPA compartments); the resulting Gibbs-Donnan effect governs the movements of both ions and water. 2 . $\mathrm{NaCl}$, in high concentration in the inner medulla conditioned by the outer medullary countercurrent processes, significantly reduces the equilibrium colloid osmotic pressure between these compartments. 3. Urea, also accumulated here by special transport mechanisms, increases the mobility of water molecules and the flexibility of the HA fibrils by loosening hydrogen bonds. These features suggest that rhythmic, small pressure increases of the pel$\mathrm{vic} /$ calyceal muscles squeeze dilute fluid out of the HA compartment and, at the same time, accelerate the outflow of fluid and albumin into the ascending vasa recta from the EPA compartment. Further, they suggest a mechanism for the phenomenon that living organisms utilize hydrostatic pressure generated by muscle contractions in water economy namely, concentrating and diluting body fluids.
\end{abstract}

Copyright $\odot 2009$ S. Karger AG, Basel

\section{KARGER}

Fax +4161306 1234 E-Mail karger@karger.ch www.karger.com

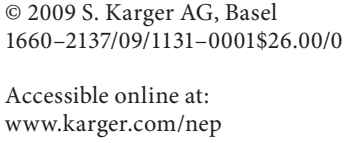

\section{Introduction}

The inner medulla of the mammalian kidney plays an important role in concentrating the urine, yet many details of the local concentrating mechanisms remain unexplained. While the mechanism of the accumulation of urea has been clarified (see review [1]), the model of the comprehensive mechanism for the concentrating process proposed independently by Stephenson [2] and Kokko and Rector [3], was not borne out by experimental results [4]. In this paper we focus on the observations of SchmidtNielsen and coworkers [5-8] that absence of rhythmic contractions of the renal pelvic muscles is associated with a decrease of the solute concentration in the inner medulla. Knepper et al. [9] proposed that a macromolecule, hyaluronan (HA), played the role of a mechano-osmotic transducer in the process. In an earlier paper [10] we discussed the role of macromolecules in the inner medulla and argued that by considering the thermodynamic equivalence between mechanical and osmotic work, the work exerted by the pelvic muscles seemed disproportionately small to account for the increase of solute concentration occurring in the inner medulla. The hypothesis developed here provides a possible resolution of this discrepancy.

We have previously proposed [10] that in the inner medullary interstitium of the mammalian kidney there are 2 fluid compartments: the HA compartment and the extravasated plasma albumin (EPA) compartment. Although these compartments are not separated by a mem-

G.G. Pinter

9321 Dunloggin Road

Ellicott City, MD 21042 (USA)

Tel. +1 410461 3740, E-Mail ggvp@comcast.net 
brane, the separation is the result of molecular exclusion, a phenomenon described by Ogston, Laurent and associates [11-13] as it relates specifically to hyaluronan and albumin. Distribution of ions and water between these compartments is determined by the Gibbs-Donnan relationship.

In the Gibbs-Donnan model the charged colloid necessitates a redistribution of both positive and negative small ions in order to approach electroneutrality between 2 compartments on both sides of a membrane which is permeable to water and small ions but not to colloid molecules. The movement of ions causes osmotic imbalance and consequent water movement that, in turn, disturbs the balance in ion concentrations, whereupon further ion migration takes place. In the absence of an external intervention, relocation of ions and osmotic water redistribution would continue until the compartment containing the charged colloid would absorb practically the entire other compartment. In models of Gibbs-Donnan equilibrium, such external intervention is usually applied as a hydrostatic pressure on the colloid-containing compartment. At equilibrium the excess hydrostatic pressure on the colloid-containing side imparts an increase of water potential which is sufficient to balance the higher potential of water on the other side, so that no net movement of water occurs between the 2 compartments.

The interstitial space in the renal inner medulla is about $10-15 \%$ of the total tissue volume which is larger than the interstitial space in other regions of the renal tissue [14]. The HA content of the inner-medullary interstitium averages approximately $1.0 \mathrm{mg} / \mathrm{ml}$ wet weight of tissue and shows an increasing concentration profile toward the papilla $[15,16]$. The extravasated albumin molecules are mobile and, driven by solvent drag, move rapidly through their EPA compartment and re-enter into the ascending vasa recta $[17,18]$. We estimated $[10]$ that the EPA compartment constitutes approximately $15-20 \%$ of the interstitial volume, and cited experimental data $[19$, $20]$ indicating that the concentration of albumin molecules in this compartment is about one third to one half of that in circulating plasma.

The HA and the EPA compartments together constitute a complex interspersed structure. The interwoven HA molecular fibrils are surrounded by sheets of water in which the mobility of water molecules is restricted [21]. The Gibbs-Donnan relationship, which influences the movements of water and small ions between the HA and EPA compartments, is modulated by the presence of $\mathrm{NaCl}$. Urea and $\mathrm{NaCl}$ are approximately equal constituents responsible for the high osmolality of the inner med- ullary tissue [22] which rises steeply toward the papilla, and reaches its highest value well in excess of $1 \mathrm{osm} / \mathrm{kg}$ of water in many species.

\section{Modulated Colloid Osmotic Effect}

In their early paper, Donnan and Harris [23], using Congo-red as a negatively charged non-penetrating colloid, reported that the colloid osmotic pressure was progressively diminished with increasing $\mathrm{NaCl}$ concentration. An explicit formulation of the quantitative effect of $\mathrm{NaCl}$ concentration on equilibrium colloid osmotic pressure was derived by Hitchcock [24] from Donnan's theory of equilibrium which is based on the requirement that the concentrations of diffusible ions at equilibrium conform to the equality of ion products. Derivation of the formula is found in the Appendix. The formula reads:

$$
\mathrm{P}=R T\left[\mathrm{z} / \mathrm{n}+\left(\mathrm{z}^{2}+4 \mathrm{x}^{2}\right)^{1 / 2}-2 \mathrm{x}\right]
$$

where $\mathrm{P}$ is the equilibrium colloid osmotic pressure in $\mathrm{mm} \mathrm{Hg} ; R$ is the gas constant in appropriate dimensions and $T$ the absolute temperature in Kelvin; $\mathrm{z}$ represents the molal-equivalent concentration of the colloidal anion, $n$ is the number of negative charges of one colloidal molecule, and $\mathrm{x}$ is the equilibrium equivalent concentration of the diffusible $\mathrm{Na}$ and also the $\mathrm{Cl}$ ions, each, in the solution on the side of the membrane that does not contain the charged colloid, where their concentrations are equal.

By using Hitchcock's formula, we calculated the approximate effects of varying $\mathrm{NaCl}$ concentrations on the equilibrium colloid osmotic pressure developed by a colloidal solution of a linear polymer of the size of $10^{6} \mathrm{Dal}-$ tons at a concentration of $1 \mathrm{~g} / \mathrm{kg}$ of water, at $38^{\circ} \mathrm{C}$. The osmotic pressure for such a solution, if the colloidal molecule is assumed to carry no electric charge and if no swelling pressure is to be taken into account, is less than 0.02 $\mathrm{mm} \mathrm{Hg}$. When using the above formula for charged colloids, constant $\mathrm{pH}$ was assumed. The calculations showed that the size of the colloid (up to 10 million Daltons) and its concentration (up to $10 \mathrm{~g} / \mathrm{kg}$ of water) had only unremarkable effects on the equilibrium colloid osmotic pressure.

As shown on figure 1, both the electric charge density of the colloid molecule and the $\mathrm{NaCl}$ concentration are predicted to have substantial effects on the equilibrium colloid osmotic pressure. The 3 different lines on the graph represent 3 different charged states of the colloidal molecule such that every one of the one-million-Dalton- 


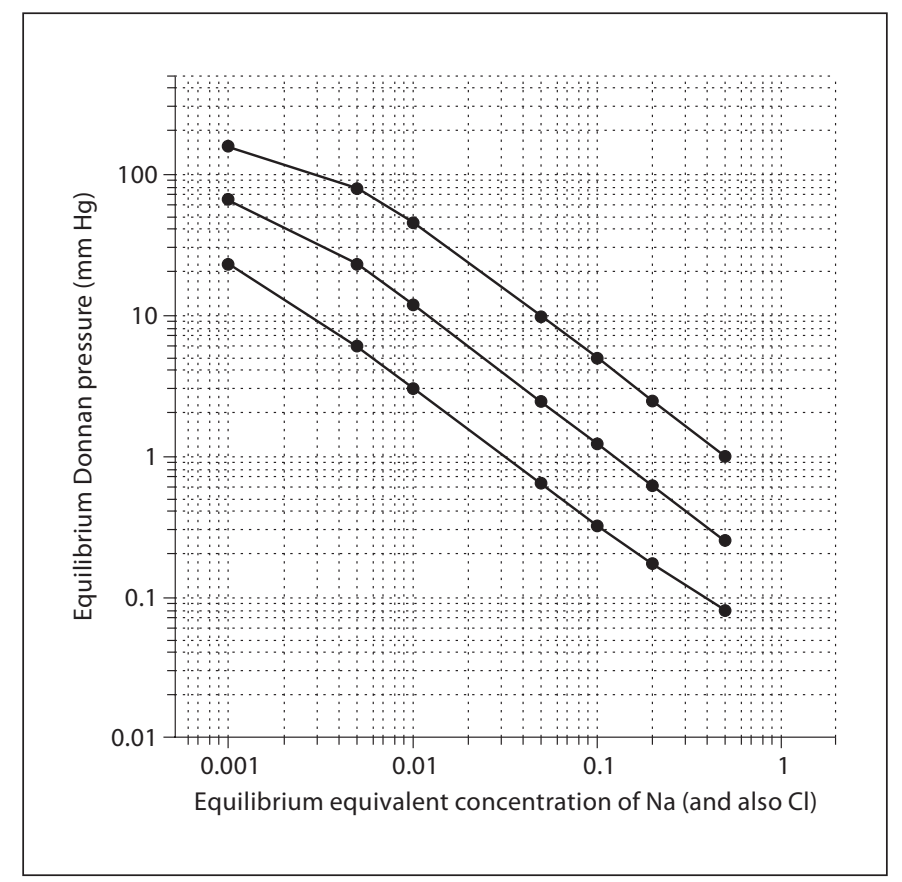

Fig. 1. Calculated effects of $\mathrm{Na}$ concentration on the equilibrium Donnan pressure of 3 generic colloids of $10^{6}$ Dalton MW each of different charge densities at a concentration of $1 \mathrm{~g} / \mathrm{kg}$ of water. Three different lines represent 3 different charged states: each molecule carries, on the top line: 10,000 middle line: 5,000; lowest line: 2,500 unit electric charges. Log-log coordinates. For further details, see text.

size colloid molecule carries 10,000 negative unit charges on the top line, 5,000 charges on the middle line and 2,500 charges on the bottom line, (i.e. in the polymer each respective 100, 200, and 400 Dalton size repeating subunit carries one unit negative charge). No internal interference of these charges is assumed. We refer to these as high, moderate, and low charged states, respectively.

According to Laurent and Fraser [13] HA is a linear polymer built from repeating disaccharide units of Dglucuronic acid - N-acetyl-D-glucosamine. Providing that each of these, 405 Dalton size, repeating disaccharide unit carries 1 free negative charge of a carboxyl group, the charge density of the HA molecule should be close to the low-charged generic polymer represented in the Figure.

On the abscissa in figure 1, the equilibrium equivalent concentration of $\mathrm{Na}$ (and also $\mathrm{Cl}$ ) on the side that does not contain colloid are marked, vs. the equilibrium colloid osmotic pressure on the ordinate. The figure shows that when the concentration is $0.001 \mathrm{Na} \mathrm{Eq} / \mathrm{kg}$, the equilibrium Donnan pressure generated by the highly charged colloidal solution is $159 \mathrm{~mm} \mathrm{Hg}$. For the moderately charged colloid it is $66 \mathrm{~mm} \mathrm{Hg}$, and about $23 \mathrm{~mm} \mathrm{Hg}$ for the low charged state of the colloid. The figure also shows that with increasing $\mathrm{Na}$ concentration the equilibrium colloid osmotic pressure is drastically diminished in all cases, to such a degree that when the equilibrium $\mathrm{NaCl}$ concentration on the side not containing colloid is 0.2 $\mathrm{Eq} / \mathrm{kg}$, (approximately at mark 0.1 on the $\mathrm{X}$ axis) the low charged colloid develops a Donnan pressure of less than $1 \mathrm{~mm} \mathrm{Hg}$; even the colloid carrying four times as many charges generates approximately $3 \mathrm{~mm} \mathrm{Hg}$ pressure.

While the above-presented calculations relate to a generic charged polymer, the broad ranges of assumed charge densities, molecular sizes and concentrations show the basic idea of our hypothesis, which is that owing to the presence of high $\mathrm{NaCl}$ concentration in the inner medulla, the osmotic pressure generated between the HA and EPA compartments should be reduced to very low levels so that it is balanced and can be exceeded by low hydrostatic pressures. As Hitchcock's formula shows, with increasing $\mathrm{NaCl}$ concentrations, the values of the colloid osmotic pressure asymptote to the value of the osmotic pressure generated by the colloid alone. While these pressures are very low, they are always positive. Thus, without an intervening event, water will tend to enter into the HA compartment however high the $\mathrm{NaCl}$ concentration may be. The events that intervene are the cyclic pressure waves generated by the pelvic/calyceal muscles.

\section{Working Hypothesis}

According to our hypothesis, in the HA compartment of the inner medullary interstitium, the charged HA molecules, jointly with the $\mathrm{Na}$ and $\mathrm{Cl}$ ions, develop a colloid osmotic pressure and absorb ions and water from the EPA compartment. The EPA compartment is supplied with fluid and elevated $\mathrm{NaCl}$ concentration (owing to the countercurrent mechanisms operating in the outer medulla) from the descending loops of Henle and vasa recta. In the concentrating kidney, a small amount of water also enters from the collecting ducts. We suggest that uptake of fluid by the HA compartment is cyclically interrupted and reversed by pressure increases exerted by the pelvic muscles (in monopyramidal kidneys) or the calyceal muscles (in multipyramidal kidneys); these respective parts at the beginning of the ureter enfold the inner medulla and the papilla. The hydrostatic pressure generated by these muscles has not been measured to our knowledge. An estimate for the calyceal pressures in man may 
be obtained from the studies of Kiil [25]. This author inserted a catheter in a human patient from the ureter into a calyx, and after finding no muscle activity, injected a few milliliters of fluid (containing X-ray contrast material) and elicited cyclic contractions of the calyceal muscles. The peak magnitude of the pressure waves can be estimated from his figure 27 (on p.92 of ref. 25) to be approximately $10 \mathrm{~mm} \mathrm{Hg}$.

To be effective in squeezing fluid from the HA compartment, the hydrostatic pressure has to exceed the Donnan pressure which, as shown above, at $\mathrm{NaCl}$ concentrations prevalent in most mammalian inner medullae, is very low. The pressure has to overcome also the rigidity of the HA chains. As shown by Gribbon et al. [26], in this the high concentration of urea in the inner medulla should play an important role. These authors stated '... urea increases the flexibility of HA chains. The primary intramolecular chain-stiffening mechanism for $\mathrm{HA}$ arises from hydrogen-bonding between adjacent saccharides. ... (Urea) ... disrupt(s) only a sub-fraction of hydrogen bonds such as those involving a water bridge.' The urea concentration producing this effect was relatively low: it was present at $0.5 \mathrm{M}$ and higher concentrations, at levels which are in the range found in the mammalian inner medulla.

The pressure exerted by calyceal/pelvic muscle contractions should affect both the HA and the EPA compartments. The main effect of the increased pressure on the EPA compartment would enhance fluid outflow toward its sink, i.e. into the ascending vasa recta.

The maintenance of material balance and the concentrating function should depend on the particular fact that when squeezed, the HA compartment would not act as a simple sponge. The composition of the fluid squeezed out of a simple sponge is the same as the fluid in the inner labyrinth of the sponge. In contrast, when the HA compartment is squeezed, at first a relatively dilute fluid would be removed from it. This is because, although there is no membrane between the HA and EPA compartments, in the HA compartment ions, other solutes, and water are held together by various physical and chemical bonds within and around the interlaced fibrillar network of the HA molecules. When subjected to a transient squeeze, at first dilute fluid would be removed from the HA compartment because water molecules are less strongly bound than $\mathrm{Na}^{+}$ions which are more strongly tied to the HA reticulum. Hence, the importance of the pulsatile nature of the pressure, as a prolonged squeeze would remove fluid with increasing solute concentration. In the relaxation phase, the HA compartment would be reconstituted by absorbing both lost water and ions, whereby the conditions prior to the squeeze should be restored. As noted by $\mathrm{Lu}$ et al. [27] in a similar system, over a few cycles this type of a process tends towards dynamic steady state.

\section{Discussion}

The macromolecular components of this hypothesis have been subject of extensive research in times earlier. The high concentration of HA in the inner medulla of the mammalian kidney has been described and suggestions have been made for its possible role in the urine concentrating process by several authors [28-30]. Knepper et al. [9] presented an insightful conjecture for the role of HA as a transducer of pressure energy into osmotic work. Our conceptual difficulty with the quantitative aspect of their conjecture contributed part of the impetus for the macromolecular hypothesis presented in this paper.

The large quantity of extravasated albumin in the renal interstitium has been first noted by Pappenheimer and Kinter [31] and also by Weaver et al. [32]. A possible connection between extravascular albumin and the urine concentrating process has been described by Pinter [19] who found a significant correlation between the urine osmolality and the extravascular albumin distribution space in the canine medulla, but no explanation for this finding was apparent. Wilde and Vorburger [33] discussed the interaction between the accumulation of extravascular albumin by means of an 'albumin multiplier' and the entry of Na into the inner medullary interstitium. MacPhee [16] concluded that both HA and extravasated albumin were involved in the inner medullary concentrating process, but the mechanism of this effect was not specified.

\section{Conclusion}

This proposed hypothesis embraces most of the known structural and functional characteristics of the inner medulla of the mammalian kidney. It takes into account the macromolecular composition, the high concentrations of $\mathrm{NaCl}$ and urea, the relatively large size of the interstitium, and the rhythmic contractions of the pelvic muscles. As described in more details above, this proposed hypothesis of a concentrating process depends on the negatively charged colloidal HA in the HA compartment which, together with the ions, generates a colloid osmotic pressure 
absorbing water and ions. Owing to the relatively high concentration of $\mathrm{NaCl}$, this colloid osmotic pressure is reduced to low levels so that it would be cyclically stopped and reversed by small hydrostatic pressures which are generated by the pelvic/calyceal muscle contractions. These contractions squeeze fluid from the HA compartment. The first portion of squeezed-out fluid is dilute; subsequently - depending on the duration of the squeeze - it should contain an increasing concentration of ions. In the muscle relaxation phase the uptake of fluid by the HA compartment would be restored.

When, in the concentrating kidney, a small amount of excess water is added to the fluid absorbed by the HA compartment, alterations of pressure, duration and frequency of muscle contractions or a combination of these can adjust to the perturbation. In water diuresis when the aquaporine channels of the collecting duct wall are not open, the dilute tubular fluid from the distal convolutions flowing through the collecting ducts is further diluted by reabsorption of solutes, resulting in the excretion of dilute urine. Under the latter conditions the pulsatile cycles would continue to be instrumental in the material turnover and the recharging of the effectiveness of the HA compartment.

We have not attempted to incorporate this proposed mechanism as a single effect into a countercurrent multiplication scheme. The increasing concentration of HA [16] may help explain, at least partially, a similar gradient of $\mathrm{Na}^{+}$ions.

A model of water conservation of the mammalian kidney by countercurrent flows was first proposed around the middle of the last century by Kuhn and Ryffel [34]. Schmidt-Nielsen and her associates [5-8] described that muscle contractions are involved in water metabolism in the mammalian kidney and also in other species, namely insects, and proposed a hypothesis about a concentrating mechanism that involves hydrostatic pressure generated by muscle contractions [6]. Her work and our hypothesis of a macromolecular mechanism should direct further attention to this hitherto little explored mechanism of water conservation in living organisms.

\section{Appendix}

Derivation of the Equation for Calculating Colloid Osmotic Pressure in the Gibbs-Donnan Model

In the Gibbs-Donnan model, a membrane which is not permeable to negatively charged colloids but permeable to $\mathrm{Na}^{+}$and $\mathrm{Cl}^{-}$ ions separates two solutions. One solution contains a charged colloid $\mathrm{R}^{\mathrm{n}-}$ at a concentration of $\mathrm{z} / \mathrm{n}$, where $\mathrm{z}$ is the molal concentration of the colloidal anion, plus $\mathrm{Cl}$ ions at the equilibrium molal concentration of $\mathrm{y}$, plus $\mathrm{Na}$ ions at the equilibrium molal concentration of $y+z$. The solution on the other side of the membrane contains $\mathrm{Na}$ and $\mathrm{Cl}$ ions both at the molal concentration of $\mathrm{x}$. The osmotic pressure is generated by the difference between the sums of molecular concentrations of all solutes in both sides, and the equilibrium state is maintained by exerting a hydrostatic pressure of $\mathrm{P}(\mathrm{mm} \mathrm{Hg})$ on the colloid side. Italicized $R$ and $T$ denote the gas constant (in appropriate dimension) and the absolute temperature (in centigrade). Thus:

$$
\mathrm{P}=R T[(\mathrm{z}+\mathrm{z} / \mathrm{n}+2 \mathrm{y})-2 \mathrm{x}]
$$

According to Donnan's theory of membrane equilibrium, the concentrations of diffusible ions conform to the ion product equation:

$$
\mathrm{x}^{2}=\mathrm{y}(\mathrm{y}+\mathrm{z})
$$

If $y$ is expressed from equation (2) and substituted into equation (1), we get the working equation:

$$
\mathrm{P}=R T\left[\mathrm{z} / \mathrm{n}+\left(\mathrm{z}^{2}+4 \mathrm{x}^{2}\right)^{1 / 2}-2 \mathrm{x}\right]
$$

Assumptions. No swelling pressure; no interacting charges on the colloid; the osmotic coefficient of all solutes is unity.

\section{References}

1 Yang B, Bankir L: Urea and urine concentrating ability: new insights from studies in mice. Am J Physiol Renal Physiol 2005;288: F881-F896.

2 Stephenson JL: Concentration of urine in a central core model of the renal counterflow system. Kidney Int 1972;2:85-94.

3 Kokko JP, Rector FC Jr: Countercurrent multiplication system without active transport in inner medulla. Kidney Int 1972;2:214223.
4 Layton HE, Knepper MA, Chou CL: Permeability criteria for effective function of passive countercurrent multiplier. Am J Physiol Renal Physiol 1996;270:F9-F20.

5 Schmidt-Nielsen B: Function of the Renal Pelvis; in Kinne RKH, Kinne-Safran E, Beyenbach KW (eds): Comparative Physiology. New York, Karger, 1990, pp 103-140.

6 Schmidt-Nielsen B: The renal concentrating mechanism in insects and mammals: a new hypothesis involving hydrostatic pressures. August Krogh Lecture. Am J Physiol Reg Integr Comp Physiol 1995;268:R1087-R1100.
7 Dwyer TM, Schmidt-Nielsen B: The renal pelvis: machine that concentrates urine in the papilla. News Physiol Sci 2003;18:1-6.

$>8$ Pruitt MEC, Knepper MA, Graves B, Schmidt-Nielsen B: Effect of the contractions of the renal pelvic wall on solute concentrations of the renal inner medulla in the hamster. Am J Physiol Renal Physiol 2006; 290:F892-F896. 
9 Knepper MA, Saidel GM, Hascall VC, Dwyer T: Concentration of solutes in the renal inner medulla: interstitial hyaluronan as a mechano-osmotic transducer. Am J Physiol Renal Physiol 2003;284:F433-F446.

$\checkmark 10$ Pinter GG, Shohet JL: Two fluid compartments in the renal inner medulla: a view through the keyhole of the concentrating process. Phil Trans R Soc A 2006;364:15511561.

$>11$ Ogston AG, Phelps CF: The partition of solutes between buffer solutions and solutions containing hyaluronic acis. Biochem J 1960; 78:827-833.

$>12$ Laurent TC, Ogston AG: The interaction between polysaccharides and other macromolecules. 4. The osmotic pressure of mixtures of serum albumin and hyaluronic acid. Biochem J 1963;89:249-253.

13 Laurent TC, Fraser RE: Hyaluronan. The FASEB Journal 1992;6:7397-7404.

14 Langer KH: Renal Interstitium Ultrastructure and Capillary Permeability; in Maunsbach AB, Olsen TS, Christensen I (eds): Functional Ultrastructure of the Kidney. New York, Academic Press, 1980, pp 431442 .

-15 Castor CW, Green JA: Regional distribution of acid mucopolysaccharides in the kidney. J Clin Invest 1968;47:2125-2132.

$\checkmark 16$ MacPhee PJ: Estimating the renal medullary interstitial oncotic pressures and the driving force for fluid uptake into ascending vasa recta. J Physiol 1998;506:529-538.

17 MacPhee PJ, Michel CC: Fluid uptake from the renal medulla into ascending vasa recta in anaesthetized rats. J Physiol 1995;487: 169-183.
8 Tenstad O, Heyeraas KJ, Wiig H \& Aukland $\mathrm{K}$ : Drainage of plasma proteins from the medullary interstitium in rats. J Physiol 2001;536:533-539.

19 Pinter GG: Distribution of chylomicrons and albumin in dog kidney. J Physiol 1967;192: 761-772

20 Pallone TL: Extravascular protein in the renal medulla: analysis by two methods. Am J Physiol Regul Integr Comp Physiol 1994; 266:R1429-R1436.

21 Cowman MK, Liu J Li, Hitcher DM, Kim JS Hyaluronan interactions: self, water, ions; in Laurent TC (ed): The Chemistry, Biology, and Medical Applications of Hyaluronan and its Derivatives. London, Wenner-Gren International Series, Portland Press, 1998, vol. 72, pp.17-24.

-22 Ullrich KJ, Jarausch KH: Untersuchungen zum Problem der Harnkonzentrierung und Harnverdünnung. Über die Verteilung von Elektrolyten ( $\mathrm{Na}, \mathrm{K}, \mathrm{Ca}, \mathrm{Mg}, \mathrm{Cl}$, anorganischem Phosphat), Harnstoff, Aminosäuren und exogenem Kreatinin in Rinde und Mark der Hundeniere bei verschiedenen Diuresezuständen. Pflügers Arch 1956;262: 537-550.

23 Donnan FG, Harris AB: The osmotic pressure and conductivity of aqueous solutions of Congo-red, and reversible membrane equilibria. Journal of the Chemical Society, Transactions 1911;99:1554-1577.

24 Hitchcock DI: Some consequences of the theory of membrane equilibria. J Gen Physiol 1925;9:97-109.

25 Kiil F: The Function of the Ureter and Renal Pelvis. Pressure Recordings and Radiographic Studies of the Normal and Diseased Upper Urinary Tract. Philadelphia, WB Saunders, 1957.
26 Gribbon P, Heng BC, Hardingham TE: The analysis of intermolecular interactions in concentrated hyaluronan solutions suggest (sic) no evidence for chain-chain association. Biochem J 2000;350:329-335.

$27 \mathrm{Lu}$ Y, Parker KH, Wang W: Effects of osmotic pressure in the extracellular matrix on tissue deformation. Phil Trans R Soc A 2006; 364:1407-1422.

28 Koefoed J, Knudsen PJ: Countercurrent concentration by a colloid degradation water pump. Proc. First Internat. Congress of $\mathrm{Ne}$ phrology, Basel, Karger, 1961, pp 571-573.

29 Pinter GG: A possible role of acid mucopolysaccharides in the urine-concentrating process. Experientia 1967;23:100.

30 Morard JC: Études histochemiques sur le role des mucopolysaccharides de la médullaire rénal dans les processus de la concentration urinaire. CR Acad Sci 1967;264: 2166-2169.

-31 Pappenheimer JR, Kinter WB: Hematocrit ratio of blood within mammalian kidney and its significance for renal hemodynamics. Am J Physiol 1956;185:377-390.

32 Weaver AN, McCarver CT, Swann HG: Distribution of blood in the functional kidney. J Exp Med 1956;104:41-55.

33 Wilde WS, Vorburger C: Albumin multiplier in kidney vasa recta analyzed by microspectrophotometry of T-1824. Am J Physiol 1967; 213:1233-1243.

34 Kuhn W, Ryffel K: Herstellung konzentrierter Lösungen aus verdünnten durch blosse Membranwirkung. Ein Modellversuch zur Funktion der Niere. Z Physiol Chem 1942; 276:145-157. 